\title{
Autologous fat grafting in the face and neck-Multinational trends and knowledge of the safety, applications and indications considering oncologic risk potential
}

\author{
Lukas S Fiedler \\ Daniel B Saleh \\ Alicia Mukrowsky
}

\begin{abstract}
Introduction Autologous fat grafting (AFG) has both aesthetic and reconstructive applications. Described by Neuber in 1893 there are numerous applications of $A F G$ as the body of evidence for its use has expanded. The earliest controversies were evident in lipofilling for breast defects post-oncological treatment, and to this day some countries do not allow it for fear of inducing tumourogenesis in an oncologically ablated field. Adipose-derived stem cells (ASCs) have been shown pro-oncologic behaviour in xenografts, however, this has not been reproduced in clinical observations thus far.

Material and Methods We sought to review contemporary harvesting and processing techniques for $A F G$ in the craniofacial region, therefore distributed a survey to evaluate the opinion and clinical impact of oncological risk across four European countries with possibly differing clinical attitudes.

Results In the craniofacial region $A F G$ is mostly used for aesthetic indications, cannula hand-held aspiration and centrifugation seem to be the predominant harvesting and processing techniques. Half of our respondents tell patients about the possibility of pro-oncologic behaviour of $A F G$. We found no significant geographical differences between the German-speaking and the English groups concerning their harvesting and processing technique. German participants seem to perform $A F G$ more often and mostly use it for cosmetic and post-cancer treatment sequelae, English participants homogenically use $A F G$ for cosmetic reasons.
\end{abstract}

Conclusion Theoretical pro-oncologic behaviour of craniofacial $A F G$ does not deter surgeons. Perhaps, we shouldn't consider discussing this complex area of oncogenesis with patients as there is no elevated risk from what we know. 


\begin{abstract}
Introduction Autologous fat grafting (AFG) has both aesthetic and reconstructive applications. Described by Neuber in 1893 there are numerous applications of $A F G$ as the body of evidence for its use has expanded. The earliest controversies were evident in lipofilling for breast defects post-oncological treatment, and to this day some countries do not allow it for fear of inducing tumourogenesis in an oncologically ablated field. Adipose-derived stem cells (ASCs) have been shown pro-oncologic behaviour in xenografts, however, this has not been reproduced in clinical observations thus far.

Material and Methods We sought to review contemporary harvesting and processing techniques for $A F G$ in the craniofacial region, therefore distributed a survey to evaluate the opinion and clinical impact of oncological risk across four European countries with possibly differing clinical attitudes.

Results In the craniofacial region $A F G$ is mostly used for aesthetic indications, cannula hand-held aspiration and centrifugation seem to be the predominant harvesting and processing techniques. Half of our respondents tell patients about the possibility of pro-oncologic behaviour of $A F G$. We found no significant geographical differences between the German-speaking and the English groups concerning their harvesting and processing technique. German participants seem to perform $A F G$ more often and mostly use it for cosmetic and post-cancer treatment sequelae, English participants homogenically use $A F G$ for cosmetic reasons.
\end{abstract}

Conclusion Theoretical pro-oncologic behaviour of craniofacial $A F G$ does not deter surgeons. Perhaps, we shouldn't consider discussing this complex area of oncogenesis with patients as there is no elevated risk from what we know.

\title{
Level of evidence V.
}

\section{Introduction}

Autologous fat grafting $A F G$ is used both in aesthetic and reconstructive surgery. Neuber described fat transfer in retractile scarring and published successful outcomes (1). In recent decades this technique has been used increasingly since the development of modern liposuction techniques in the 1980 s by Illouz $(2,3)$. The technique of harvesting and processing, expanded applications and fat transfer is now commonly used in the head/face and neck. $(4,5)$ Coleman provided a comprehensive description of techniques in the 1990s, and in recent years the scientific basis of the regenerative effects of fat grafts is progressing $(6,7)$.

Adipose tissue has notable plasticity and has endocrine function.(8) A lipoaspirate of fat tissue contains an aqueous fraction, the stromal vascular fraction (SVF), which is a combination of pre-adipocytes, endothelial precursor cells, endothelial cells, macrophages, smooth muscle cells, lymphocytes, pericytes, and adipose-derived stem cells ASCs.(9) The latter have the potential to differentiate into numerous cell lines comparable with mesenchymal stem cells $(9,10)$. ASCs are involved in biologic pathways of inflammation and tumour environment.(7) ASCs have been ascertained to promote angiogenesis and further showed elevated pro-oncologic behaviour in xenografts $(10,11)$. The applications also have expanded beyond physical 'contouring and space-filling' to regenerative applications that address radiation injury, abnormal scarring, improving aged skin and managing varieties of skin injury such as burns $(2,9,12)$.

The worldwide incidence of head and neck cancer (HNC) is more than 550,000 cases with around 300,000 annual deaths. About $90 \%$ of all head and neck cancers are squamous cell carcinomas (HNSCC). HNSCC is the sixth leading cancer by incidence worldwide. $(13,14)$ About one third of these patients present with low stage disease (T1-2, N0), therefore radiation or surgery protocols are available for treatment. Higher stage disease in $H N C$ requires postoperative chemoradiotherapy (13) resulting in treatment sequelae like a cosmetic burden (15), trismus, radiotherapy-induced neck fibrosis (16) and radiodermatitis, further skin irregularities, and lymphedema (17). $A F G$ can address these complications after successful treatment. The safety of $A F G$ is largely accepted, but attitudes differ where and the anatomical region to be treated has previously been ablated for cancer(8).

Unlike breast surgery, it is not clear what current attitudes and trends are in facial fat grafting in the post-cancer treatment patient, for example, post-radiation injury, contour defect, or chronic neck lymphedema. We aimed to try ascertaining attitudes in this respect and whether these attitudes are reflected in the evidence base for these procedures. We recognize there are no English or German published guidance, healthcare system based or otherwise, to guide the treating teams.

Autologous fat grafting $A F G$ is used both in aesthetic and reconstructive surgery. Neuber described fat transfer 
in retractile scarring and published successful outcomes (1). In recent decades this technique has been used increasingly since the development of modern liposuction techniques in the 1980 s by Illouz $(2,3)$. The technique of harvesting and processing, expanded applications and fat transfer is now commonly used in the head/face and neck. Coleman provided a comprehensive description of techniques in the 1990s, and in recent years the scientific basis of the regenerative effects of fat grafts is progressing (4).

Adipose tissue has notable plasticity and has endocrine function. This type of tissue consists of different cell types; adipocytes, connective tissue, nerve tissue, stromal vascular cells and immune cells, and adipose-derived stem cells ASCs. The latter have the potential to differentiate into numerous cell lines (5). These can be endothelial cells, vascular complexes, and stromal cells. ASCs are involved in biologic pathways of inflammation and tumour environment. ASCs have been ascertained to promote angiogenesis and further showed an elevated pro-oncologic behaviour in xenografts $(5,6)$. The applications also have expanded beyond physical 'contouring and space filling' to regenerative medicine applications that address radiation injury, abnormal scarring, improving aged skin and managing varieties of skin injury such as burns $(2,7)$.

The safety of autologous fat grafting is largely accepted, but attitudes differ where and the anatomical region to be treated has previously been ablated for cancer(8).

Unlike breast surgery, it is not clear what current attitudes and trends are in facial fat grafting in the post-cancer treatment patient, for example post radiation injury, contour defect, or chronic neck lymphedema. We aimed to try ascertaining certain attitudes in this respect and whether these attitudes are reflected in the evidence base for these procedures. We also recognize there are no English or German published guidance, healthcare system based or otherwise, to guide the treating teams.

\section{Materials and Methods}

\section{Autologous fat graftingApplications and Indications of autologous fat grafting}

Fat grafting has a number of monikers and this paper will use the term $A F G$. Applications of $A F G$ are broad and treatable regions are various. A variety of clinical problems have been treated using fat, including post oncological contour defects, scar therapy, connective tissue disease, post-radiation skin injury, chronic lymphoedema, facial and body asymmetries of the soft tissues and in complex/simple burns $(12,18)$. Ultrastructural analysis of radio-damaged tissue showed a significant reduction of the capillary bed, where $A F G$ has shown to enhance tissue hydration, the induction of blood vessel formation and increased local regeneration of damaged tissue (19). Furthermore, $A F G$ can be of use in the treatment of the microangiopathic patient $(19,20)$. Autologous fat grafting, therefore, is beneficial for patients with volume loss due to the natural process of ageing, disease, trauma, congenital defects and it improves skin quality, tissue quality and scars and further gives enlarged volume in the treated region (21).

In head and neck cancer $H N C$, squamous cell carcinoma $H N S C C$ is the most common and frequently requires surgical ablation plus radiotherapy. This oncologic treatment can often lead to major functional impairment, lymphoedema and radiation-induced soft tissue changes inducing neck lymphedema and radiation injury and a restriction of function, and in facial cancer ablation, contour defects $(22,23)$.

Fat grafting first gathered prominence in the aesthetic area(24), and subsequent study showed it had a favourable complication profile compared to synthetic filler materials $(25,26)$.

However, there remains a weak consensus on the optimal conditions of fat aspiration, processing, and injection that confers a greater 'take' in aesthetic applications (25, 27-29). Further, the amount of infiltrated fat in the face is not yet standardized. (24) Moreover, due to the chronicity of radiation injury in $H N C$ treatment, the optimal time of treatment is not clear, and what is the exact change in the irradiated skin micro-environment $(2,30)$.

\section{Risks of autologous fat grafting}

Fat grafting does carry a risk of intra-luminal injection causing necrosis and infarct of the end organ tissue, which in the head and neck can be devastating and there are numerous reports of blindness, facial skin loss, fat embolic syndrome and cerebral infarct (37-39).

Donnenberg et al suggested that ASCs support growth in active breast cancer cells. In the conclusion of their xenograft study, $A F G$ in breast reconstruction should be deferred until cancer remission is confirmed. However, 
fat grafting and ASCs have not been shown to drive cancer growth in clinical reality, several studies with big cohorts showed no evidence for an elevation in oncological risk in $A F G$ (40-42).

Silva et al found no evidence for clinically relevant elevations in tumour size, proliferation, histologic grade or metastasis in $A F G$ breast reconstruction in an animal model (43). Moreover, Mazur et al found no indication for a higher breast cancer risk in ASCs augmented fat in post-cancer mastectomy and radiation patients (44).

This is sometimes a result of graft retention or simple distribution over time within the surrounding tissue. Retention rates, however, stay the same in repeated injections (45). Bourne et al found that retention rates differed in smokers, who had greater volume retention over predictable 9 months (45). Further, the study group stated that $A F G$ is less invasive and therefore safer than conventional reconstruction options (45).

The evidence overall is lacking, and there is no specific research respectively addressing the oncologic risk profile of $A F G$ in the head, face and neck. Some publications concentrated on the general complications of $A F G$ in the head, face and neck, and stated a low rate of minor complications $(5,46,47)$. Karmali et al, in their publication, investigating outcomes of $A F G$ for head and neck reconstruction and found no evidence for an association with cancer recurrence (48).

Fat grafting does carry risk of intra-luminal injection causing necrosis and infarct of the end organ tissue, which in the head and neck can be devastating and there are numerous reports of blindness, facial skin loss, fat embolic syndrome and cerebral infarct (27-29).

Donnenberg et al suggested that ASCs support growth in active breast cancer cells. In the conclusion of their xenograft study $A F G$ in breast reconstruction should be deferred until cancer remission is confirmed. However, fat grafting and ASCs have not been shown to drive cancer growth in clinical reality, several studies with big cohorts showed no evidence for an elevation in oncological risk in $A F G$ (30-32).

Silva et al found no evidence for clinically relevant elevations in tumour size, proliferation, histologic grade or metastasis in AFGbreast reconstruction in an animal model (33). Moreover, Mazur et al found no indication for a higher breast cancer risk in ASCsaugmented fat in post-cancer mastectomy and radiation patients (34).

This is sometimes a result of graft retention or simple distribution over time within the surrounding tissue. Retention rates, however, stay the same in repeated injections (35). Bourne et al found that retention rates differed in smokers, who had greater volume retention over predictable 9 months (35). Further, the studygroup stated that $A F G$ is less invasive and therefore safer than conventional reconstruction options (35).

Evidence overall is lacking, and there is no specific research respectively addressing the oncologic risk profile of $A F G$ in the head, face and neck. Some publications concentrated on the general complications of $A F G$ in the head, face and neck, and stated a low rate of minor complications (36-38). Karmali et al, in their publication, investigating outcomes of $A F G$ for head and neck reconstruction and found no evidence for an association with cancer recurrence (39).

\section{AFG Survey}

We conducted a survey to ascertain if there are differences in surgical cultural beliefs towards the safety of fat grafting in patients treated for head, neck and facial cancer. A German online survey-softwaretool @soscisurvey.de (49) was used and distributed by email, and via national associations, to head and neck surgeons working in Germany, Switzerland, Austria and UK in the field of Plastic surgery, maxillofacial surgery and Otorhinolaryngology. The participants had the option to take the survey in German or English. Questions centred around the applications, indications, safety and their personal preferences for $A F G$ (table 1). We chiefly aimed to determine if a wider European attitude to $A F G$ in the head and neck mirrored that in breast reconstructive practices post cancer fat grafting.

Do you have experience with autologous fat grafting of the head/neck and face?

How many of these procedures do you perform annually?

Do you use fat grafting for Aesthetic facial contouring?

Do you use fat grafting for Benign acquired pathology such as HIV lipodystrophy, craniofacial anomalies, facial differences or scar

Do you use fat grafting for Post cancer facial contouring, post cancer radiation treatment for facial/neck function?

In your practice please rank the main indications for fat grafting (1-3)?

What technique do you typically use to harvest your fat? 
Do you have experience with autologous fat grafting of the head/neck and face?

How do you process the fat before you infiltrate it?

Do you discuss with patients the theory of adipose derived stem cells potentiating neoplasia in a patient treated for facial, head and Do you see an elevation of cancer risk as a possible adverse effect of autologous fat grafting in treated cancer patients?

Have you ever experienced a cancer recurrence or a newly developed cancer in a facial region, where autologous fat has been inject Are you aware of any studies or reports of cancer recurrence following fat grafting?

Table 1 AFG survey questionaire $\odot$ Soscisurvey.de, a free tool for online surveys

\section{Results}

In our survey, there were 24 German and 21 English speaking respondents.

Most of our responses came from Plastic and reconstructive surgeons [43/45; 95.5\%] (see Figure 1), two Otorhinolaryngologists participated in and completed our questionnaire.

Nearly all of the participants declared experience with autologous fat grafting $[42 / 45 ; 93.6 \%]$ whereas only three colleagues [3/45; 6.6\%] had no experience with fat grafting. As shown in Figure 2, 38 percent [17/45; 37.7\%] of the responders stated an $A F G$-use under ten times a year, nearly half $[21 / 45 ; 46.6 \%]$ stated the usage of ten to fifty times per year and 14 percent $[6 / 45 ; 13.3 \%]$ were frequent users of fat grafting $(>50 /$ year).

We observed common usage for aesthetic in $84 \%$ of respondents [38/45; 84.4\%]. Further, a representative part of the participating colleagues $[28 / 36 ; 62.2 \%]$ uses $A F G$ for benign acquired pathologies such as HIV lipodystrophy, craniofacial anomalies, facial asymmetries, or scar treatment. Of particular interest, nearly two-thirds of our sample [28/36; 62.2\%] do use $A F G$ for post-cancer facial contouring or radiation-induced fibrosis treatment for facial/neck function.

As presented in Figure 3, we asked how the participating surgeons rank the indications for $A F G$ in their practice. Based on 37 valid answers in the survey, $60 \%$ deemed "aesthetic facial contouring" as the most important $A F G$ application [22/37; 59.6\%], a further 25 percent [9/37; 24.3\%] ranked "post-cancer facial contouring, post-cancer radiation treatment for facial/neck function" their most common indication.

Concerning harvest technique (see Figure 4), the majority use Syringe aspiration with a fine needle/cannula $1 \mathrm{~mm}$ or greater [24/45; 53\%], by the use of Vacuum aspiration with low pressure [17/45; 34\%]. A few surgeons favour syringe aspiration with fine needle with a lower diameter than $0,7 \mathrm{~mm}[4 / 45 ; 9 \%]$. Interestingly, nobody of our survey cohort works with surgical excision in $A F G$ applications [0/45; $0 \%]$.

Nearly half of our colleagues process their harvested tissue by centrifugation [20/43; 46.5\%], and the remainder (54\%) had variable practices (see Table 2.)

\begin{tabular}{lll}
\hline Free Answers of processing technique & $\mathbf{n}$ & $\mathbf{\%}$-absolute \\
Sedimentation and passive separation by gravity & 6 & $16.2 \%$ \\
PureGraft & 3 & $8.1 \%$ \\
decant liquid parts-no centrifuge & 2 & $5.4 \%$ \\
Separation of fat and liquid parts in the syringe & 2 & $5.4 \%$ \\
mesh washing & 2 & $8.1 \%$ \\
Drainage of sediment and nanofat preparation & 1 & $2.7 \%$ \\
Strain and irrigate & 1 & $2.7 \%$ \\
& \\
\hline
\end{tabular}

Table2 AFG processing techniques [free text] 
As demonstrated in Figure 4, nearly half of our survey participants [20/42; 47.6\%] do not discuss the scientific conjecture around possible oncological stimulation and neoplastic potentiation by ASCs, in the fat graft matrix. This correlates with the fact that most respondents do not believe there is an elevated cancer risk with fat grafting in anatomical locations where cancer has been resected, but seemingly a proportion of these clinicians do not even consider it significant enough to discuss the controversies with patients.

Similarly, almost all respondents reported they had not observed new tumour recurrence in the fat grafted bed.

\section{Discussion}

Our survey was conducted in four countries because there are no English or German published guidance on $A F G$ in the head and neck to guide the treating teams. Most of our responses came from Plastic surgeons (95.5\%), only two Otorhinolaryngologists completed our questionnaire. This response mismatch does not correlate with the count of active surgeons in the fields. There are 1328 active colleagues in Plastic surgery listed in UK and 1498 in Germany, UK counts 1916 ENT-surgeons, whereas Germany lists 2981 active colleagues in ENT (50-52). Reasonably we assume a broader use of $A F G$ in Plastic surgery.

Most colleagues who participated in our survey (46,6\%) state a ten to fifty times a year use of $A F G$ and 14 percent stated an $A F G$-use of more than fifty times a year. The frequency of usage of $A F G$ is not yet addressed in the literature. Where the indications have expanded beyond physical 'contouring and space-filling' to regenerative applications, we expect an increase of frequency of this application in use soon $(2,9,12)$.

Although aesthetic applications of fat grafting in the head and neck seem to be more prominent (84\% of respondents), a significant body of our survey respondents use autologous fat in the post-cancer treatment setting (62\%). In our opinion, this correlates with the fact, that in recent decades $A F G$ is being used increasingly. Responsible for that is the development of modern liposuction techniques in the 1980s by Illouz $(2,3)$, further Coleman provided a comprehensive description of techniques in the 1990s $(6,7)$. While fat grafting first gathered prominence in the aesthetic area (24), and a subsequent study showed it had a favourable complication profile compared to synthetic filler materials $(25,26)$, in recent years the scientific basis of the regenerative effects of fat grafting is progressing $(6,7)$.

$H N C$ represents three percent of all malignant neoplasms, $H N S C C$ is responsible for 90 percent of these cases (14). The resulting impairments of craniofacial cancer therapy, surgical reconstruction and radiotherapy make $A F G$ a very promising and useful tool to deal with scars, irregularities and radiodermatitis and chronic radiation-induced fibrosis (32).

Due to harvesting and processing in $A F G$, the majority use syringe aspiration with a fine needle/cannula $1 \mathrm{~mm}$ or greater $(53 \%)$, and Vacuum aspiration with low pressure (34\%). This correlates with a necessary low negative pressure level in aspiration and lower shear stress for the tissue. The perception seems to be, the higher the negative pressure level of liposuction, the higher the level of shear stress to the adipose tissue, reducing fat graft survival. $(6,29,53)$

Nearly half of our colleagues process their harvested tissue by centrifugation (46.5\%), and the remainder (54\%) had variable practices like sedimentation $(16 \%)$ or pure graft $(8 \%)$. Only two colleagues use mesh washing techniques. Tuin et al discovered no superior processing technique could be identified based on clinical outcome (35). The lower count of used washing techniques, in our opinion, may be because mesh washing techniques are labour intensive and are linked with a higher loss of graft material in the process, compared to the easy to use and fast centrifugation. 
Nearly half of our survey participants do not discuss elevated cancer risk. On the other hand, only 31 percent stated knowledge of studies dealing with cancer recurrence following $A F G$. Moreover, most respondents have never confidently experienced cancer recurrence or newly developed cancer in a previously ablated region, following $A F G$. In several mastectomy studies, fat grafting and $A S C$ s have not been shown to drive cancer growth in clinical reality, several studies with big cohorts showed no evidence for an elevation in oncological risk in $A F G$ (4042). Further, studies found no significant evidence for clinically relevant elevations in tumour size, proliferation, histologic grade or metastasis in $A F G$ breast reconstruction. Most of the current studies dealing with the oncologic risk of $A F G$ refer to breast cancer, further longitudinal shared data sets would be desirable to evaluate a possible pro-oncologic behaviour of $A F G$ in the face, head and neck.

In conclusion, currently, there are no evidence-based studies which would authorize a valid recommendation due to pro-oncological risk of $A F G$ in treated $H N C$ tumour sites. There is a good case to suspect a different behaviour of ASCs in breast cancer cells versus HNSCC. Perhaps, we shouldn't consider discussing this complex area of oncogenesis with patients as there is no elevated risk from what we know. However, the bulk of data is for breast carcinoma which is a different disease to HNSCC, and different tumours could conceivably interact in a different way to ASCS.

\section{Disclosures}

The authors declared no potential conflicts of interest with respect to the research, authorship, and publication of this article.

\section{Conflicts of interest}

The authors declare that they have no conflicts of interest to disclose.

\section{Statement of human and animal rights and ethical approval}

This article does not contain any studies with human participants or animals performed by any of the authors.

\section{Informed consent}

For this type of study informed consent is not required.

\section{Contributors}

FL: writing, survey design GER, dispatch GER and analysis GER/EN

SD: survey design EN, dispatch UK, editing

MA: writing, selection of publications

\section{References}

1. Neuber F. Fettransplantation. Chir Kongr Verhandl Dsch Gesellch Chir. 1893;22:66.

2. Simonacci F, Bertozzi N, Grieco MP, Grignaffini E, Raposio E. Procedure, applications, and outcomes of autologous fat grafting. Annals of medicine and surgery (2012). 2017;20:49-60.

3. Gornitsky J, Viezel-Mathieu A, Alnaif N, Azzi AJ, Gilardino MS. A systematic review of the effectiveness and complications of fat grafting in the facial region. JPRAS open. 2019;19:87-97.

4. Baum SH, Mohr C. Autologous dermis-fat grafts in head and neck patients: Indications and evaluation in reconstructive surgery. Journal of cranio-maxillo-facial surgery : official publication of the European Association for Cranio-Maxillo-Facial Surgery. 2018;46(10):1834-42.

5. Krastev TK, Beugels J, Hommes J, Piatkowski A, Mathijssen I, van der Hulst R. Efficacy and Safety of Autologous Fat Transfer in Facial Reconstructive Surgery: A Systematic Review and Meta-analysis. JAMA facial plastic surgery. 2018;20(5):351-60. 
6. Zielins ER, Brett EA, Longaker MT, Wan DC. Autologous Fat Grafting: The Science Behind the Surgery. Aesthetic surgery journal. 2016;36(4):488-96.

7. Bertheuil N, Chaput B, Ménard C, Varin A, Laloze J, Watier E, et al. Adipose mesenchymal stromal cells: Definition, immunomodulatory properties, mechanical isolation and interest for plastic surgery. Annales de chirurgie plastique et esthetique. 2019;64(1):1-10.

8. Drochioi CI, Sulea D, Timofte D, Mocanu V, Popescu E, Costan VV. Autologous Fat Grafting for Craniofacial Reconstruction in Oncologic Patients. Medicina (Kaunas, Lithuania). 2019;55(10).

9. Bora P, Majumdar AS. Adipose tissue-derived stromal vascular fraction in regenerative medicine: a brief review on biology and translation. Stem cell research \& therapy. 2017;8(1):145.

10. Freese KE, Kokai L, Edwards RP, Philips BJ, Sheikh MA, Kelley J, et al. Adipose-derived stems cells and their role in human cancer development, growth, progression, and metastasis: a systematic review. Cancer research. 2015;75(7):1161-8.

11. Rowan BG, Lacayo EA, Sheng M, Anbalagan M, Gimble JM, Jones RK, et al. Human Adipose TissueDerived Stromal/Stem Cells Promote Migration and Early Metastasis of Head and Neck Cancer Xenografts. Aesthetic surgery journal. 2016;36(1):93-104.

12. Arno A, Smith AH, Blit PH, Shehab MA, Gauglitz GG, Jeschke MG. Stem Cell Therapy: A New Treatment for Burns? Pharmaceuticals (Basel, Switzerland). 2011;4(10):1355-80.

13. Control UfIC. HEAD AND NECK CANCER. In: Organisation WH, editor. 2014.

14. Rigoni L, Bruhn RF, De Cicco R, Kanda JL, Matos LL. Quality of life impairment in patients with head and neck cancer and their caregivers: a comparative study. Braz J Otorhinolaryngol. 2016;82(6):680-6.

15. Bonadies A, Bertozzi E, Cristiani R, Govoni FA, Migliano E. Electrochemotherapy in Skin Malignancies of Head and Neck Cancer Patients: Clinical Efficacy and Aesthetic Benefits. Acta dermato-venereologica. 2019;99(13):1246-52.

16. Baldoman D, Vandenbrink R. Physical Therapy Challenges in Head and Neck Cancer. Cancer treatment and research. 2018;174:209-23.

17. Tyker A, Franco J, Massa ST, Desai SC, Walen SG. Treatment for lymphedema following head and neck cancer therapy: A systematic review. American journal of otolaryngology. 2019;40(5):761-9.

18. Garza RM, Paik KJ, Chung MT, Duscher D, Gurtner GC, Longaker MT, et al. Studies in fat grafting: Part III. Fat grafting irradiated tissue-improved skin quality and decreased fat graft retention. Plastic and reconstructive surgery. 2014;134(2):249-57.

19. Rigotti G, Marchi A, Galiè M, Baroni G, Benati D, Krampera M, et al. Clinical treatment of radiotherapy tissue damage by lipoaspirate transplant: a healing process mediated by adipose-derived adult stem cells. Plastic and reconstructive surgery. 2007;119(5):1409-22; discussion 23-4.

20. Bernier J, Bonner J, Vermorken JB, Bensadoun RJ, Dummer R, Giralt J, et al. Consensus guidelines for the management of radiation dermatitis and coexisting acne-like rash in patients receiving radiotherapy plus EGFR inhibitors for the treatment of squamous cell carcinoma of the head and neck. Annals of oncology : official journal of the European Society for Medical Oncology. 2008;19(1):142-9.

21. Simonacci F, Bertozzi N, Grieco MP, Raposio E. From liposuction to adipose-derived stem cells: indications and technique. Acta bio-medica : Atenei Parmensis. 2019;90(2):197-208.

22. Parsons JT, Mendenhall WM, Stringer SP, Amdur RJ, Hinerman RW, Villaret DB, et al. Squamous cell carcinoma of the oropharynx: surgery, radiation therapy, or both. Cancer. 2002;94(11):2967-80.

23. Ringash J. Survivorship and Quality of Life in Head and Neck Cancer. Journal of clinical oncology : official journal of the American Society of Clinical Oncology. 2015;33(29):3322-7.

24. Shue S, Kurlander DE, Guyuron B. Fat Injection: A Systematic Review of Injection Volumes by Facial Subunit. Aesthetic plastic surgery. 2018;42(5):1261-70. 
25. Agrawal KS, Bachhav M, Naik CS, Tanwar H, Sankhe SS. Autologous Fat Transfer for Esthetic Contouring of Face in Posttraumatic Nonfunctional Maxillofacial Deformities. Craniomaxillofac Trauma Reconstr. 2016;9(2):113-20.

26. Groen JW, Krastev TK, Hommes J, Wilschut JA, Ritt M, van der Hulst R. Autologous Fat Transfer for Facial Rejuvenation: A Systematic Review on Technique, Efficacy, and Satisfaction. Plast Reconstr Surg Glob Open. 2017;5(12):e1606.

27. Gamboa GM, Ross WA. Autologous fat transfer in aesthetic facial recontouring. Ann Plast Surg. 2013;70(5):513-6.

28. Kurial P. Lipomodeling - autologous fat transfer in aesthetic surgery. Cas Lek Cesk. 2018;157(6):289-92.

29. Strong AL, Cederna PS, Rubin JP, Coleman SR, Levi B. The Current State of Fat Grafting: A Review of Harvesting, Processing, and Injection Techniques. Plast Reconstr Surg. 2015;136(4):897-912.

30. Klinger M, Caviggioli F, Klinger FM, Giannasi S, Bandi V, Banzatti B, et al. Autologous fat graft in scar treatment. The Journal of craniofacial surgery. 2013;24(5):1610-5.

31. Rosen ED, Spiegelman BM. What we talk about when we talk about fat. Cell. 2014;156(1-2):20-44.

32. Drochioi CI, Sulea D, Timofte D, Mocanu V, Popescu E, Costan VV. Autologous Fat Grafting for Craniofacial Reconstruction in Oncologic Patients. Medicina (Kaunas). 2019;55(10):655.

33. Zuk PA, Zhu M, Mizuno H, Huang J, Futrell JW, Katz AJ, et al. Multilineage cells from human adipose tissue: implications for cell-based therapies. Tissue engineering. 2001;7(2):211-28.

34. Cohen SR, Hewett S, Ross L, Delaunay F, Goodacre A, Ramos C, et al. Regenerative Cells For Facial Surgery: Biofilling and Biocontouring. Aesthetic surgery journal. 2017;37(suppl_3):S16-s32.

35. Tuin AJ, Domerchie PN, Schepers RH, Willemsen JC, Dijkstra PU, Spijkervet FK, et al. What is the current optimal fat grafting processing technique? A systematic review. Journal of cranio-maxillo-facial surgery : official publication of the European Association for Cranio-Maxillo-Facial Surgery. 2016;44(1):45-55.

36. Lee JH, Kirkham JC, McCormack MC, Nicholls AM, Randolph MA, Austen WG, Jr. The effect of pressure and shear on autologous fat grafting. Plastic and reconstructive surgery. 2013;131(5):1125-36.

37. Rai S, Marsland AM, Madan V. Facial fat necrosis following autologous fat transfer and its management. J Cutan Aesthet Surg. 2014;7(3):173-5.

38. Roshandel D, Soheilian M, Pakravan M, Aghayan S, Peyman GA. Middle Cerebral Artery, Ophthalmic Artery, and Multibranch Retinal Vessel Occlusion After Cosmetic Autologous Fat Transfer to Forehead. Ophthalmic Surg Lasers Imaging Retina. 2015;46(5):593-6.

39. Shen X, Li Q, Zhang H. Massive Cerebral Infarction Following Facial Fat Injection. Aesthetic Plast Surg. 2016;40(5):801-5.

40. Simorre M, Chaput B, Voglimacci Stephanopoli M, Garrido I, Soule-Tholy M, Leguevaque P, et al. [Lipofilling in breast reconstruction: is there any population with higher risk of local recurrence? Literature systematic review]. Gynecologie, obstetrique \& fertilite. 2015;43(4):309-18.

41. Fraser JK, Hedrick MH, Cohen SR. Oncologic risks of autologous fat grafting to the breast. Aesthetic surgery journal. 2011;31(1):68-75.

42. Calabrese C, Kothari A, Badylak S, Di Taranto G, Marcasciano M, Sordi S, et al. Oncological safety of stromal vascular fraction enriched fat grafting in two-stage breast reconstruction after nipple sparing mastectomy: long-term results of a prospective study. European review for medical and pharmacological sciences. 2018;22(15):4768-77.

43. Silva MMA, Kokai LE, Donnenberg VS, Fine JL, Marra KG, Donnenberg AD, et al. Oncologic Safety of Fat Grafting for Autologous Breast Reconstruction in an Animal Model of Residual Breast Cancer. Plastic and reconstructive surgery. 2019;143(1):103-12. 
44. Mazur S, Zolocinska A, Siennicka K, Janik-Kosacka K, Chrapusta A, Pojda Z. Safety of adipose-derived cell (stromal vascular fraction - SVF) augmentation for surgical breast reconstruction in cancer patients. Advances in clinical and experimental medicine : official organ Wroclaw Medical University. 2018;27(8):1085-90.

45. Bourne DA, Bliley J, James I, Donnenberg AD, Donnenberg VS, Branstetter BFt, et al. Changing the Paradigm of Craniofacial Reconstruction: A Prospective Clinical Trial of Autologous Fat Transfer for Craniofacial Deformities. Annals of surgery. 2019.

46. Hammond SE, Samuels S, Thaller S. Filling in the Details: A Review of Lipofilling of Radiated Tissues in the Head and Neck. The Journal of craniofacial surgery. 2019;30(3):667-71.

47. Bellini E, Grieco MP, Raposio E. The science behind autologous fat grafting. Annals of medicine and surgery (2012). 2017;24:65-73.

48. Karmali RJ, Hanson SE, Nguyen AT, Skoracki RJ, Hanasono MM. Outcomes following Autologous Fat Grafting for Oncologic Head and Neck Reconstruction. Plastic and reconstructive surgery. 2018;142(3):771-80.

49. GmbH SS. online [cited 2020]. Available from: www.soscisurvey.de.

50. DGPRAEC. Statistik 2011. Available from: https://www.dgpraec.de/presse/statistik/.

51. workforce [Internet]. NHS. 2020. Available from: https://digital.nhs.uk/data-andinformation/publications/statistical/nhs-workforce-statistics/september-2020.

52. Deutschland B. Berufsstatistik. 2018.

53. Fontes T, Brandao I, Negrao R, Martins MJ, Monteiro R. Autologous fat grafting: Harvesting techniques. Annals of medicine and surgery (2012). 2018;36:212-8. 